\title{
Application of the RIMARC algorithm to a large data set of action potentials and clinical parameters for risk prediction of atrial fibrillation
}

\author{
Ursula Ravens • Deniz Katircioglu-Öztürk • Erich Wettwer • Torsten Christ • \\ Dobromir Dobrev $\cdot$ Niels Voigt $\cdot$ Claire Poulet $\cdot$ Simone Loose $\cdot$ Jana Simon $\cdot$ \\ Agnes Stein · Klaus Matschke • Michael Knaut $\cdot$ Emre Oto $\cdot$ Ali Oto $\cdot$ H. Altay Güvenir
}

Received: 5 June 2014 / Accepted: 16 November 2014 / Published online: 3 December 2014

(C) International Federation for Medical and Biological Engineering 2014

\begin{abstract}
Ex vivo recorded action potentials (APs) in human right atrial tissue from patients in sinus rhythm (SR) or atrial fibrillation (AF) display a characteristic spike-anddome or triangular shape, respectively, but variability is huge within each rhythm group. The aim of our study was to apply the machine-learning algorithm ranking instances by maximizing the area under the ROC curve (RIMARC) to a large data set of 480 APs combined with retrospectively collected general clinical parameters and to test whether the rules
\end{abstract}

Ursula Ravens, Deniz Katircioglu-Öztürk, Ali Oto and H. Altay Güvenir have equally contributed.

Electronic supplementary material The online version of this article (doi:10.1007/s11517-014-1232-0) contains supplementary material, which is available to authorized users.

U. Ravens · E. Wettwer - T. Christ · D. Dobrev · N. Voigt ·

C. Poulet $\cdot$ S. Loose $\cdot$ J. Simon

Department of Pharmacology and Toxicology, Medical Faculty

Carl Gustav Carus, TU Dresden, Dresden, Germany

\section{U. Ravens $(\bowtie)$}

Institut für Pharmakologie und Toxikologie, TU Dresden,

Fetscherstrasse 74, 01307 Dresden, Germany

e-mail: ravens@ rcs.urz.tu-dresden.de;

ravens@mail.zih.tu-dresden.de

D. Katircioglu-Öztürk

Department of Medical Informatics, Informatics Institute, Middle

East Technical University, Ankara, Turkey

D. Katircioglu-Öztürk $\cdot$ E. Oto

MITS, Ankara, Turkey

Present Address:

T. Christ

Department of Experimental Pharmacology and Toxicology,

University Medical Center Hamburg-Eppendorf, Hamburg,

Germany learned by the RIMARC algorithm can be used for accurately classifying the preoperative rhythm status. APs were included from 221 SR and 158 AF patients. During a learning phase, the RIMARC algorithm established a ranking order of 62 features by predictive value for SR or AF. The model was then challenged with an additional test set of features from 28 patients in whom rhythm status was blinded. The accuracy of the risk prediction for AF by the model was very good (0.93) when all features were used. Without the seven AP features, accuracy still reached 0.71 . In conclusion, we have shown that training the machine-learning algorithm RIMARC with an experimental and clinical data set allows predicting a classification in a test data set with high accuracy. In a clinical setting, this approach may prove useful for finding hypothesis-generating associations between different parameters.

Present Address:

D. Dobrev $\cdot$ N. Voigt

Institute of Pharmacology, Faculty of Medicine, University

of Duisburg-Essen, Essen, Germany

Present Address:

C. Poulet

Imperial College, London, UK

A. Stein

Department of Anesthesiology, Heart Center Dresden, Dresden,

Germany

K. Matschke $\cdot$ M. Knaut

Clinic for Cardiac Surgery, Heart Center Dresden, Dresden,

Germany

A. Oto

Department of Cardiology, Hacettepe University Hospital,

Ankara, Turkey 
Keywords Atrial fibrillation - Risk prediction - RIMARC algorithm $\cdot$ Human right atrial action potentials $\cdot$ Clinical parameters

\section{Abbreviations}

\begin{tabular}{|c|c|}
\hline $\mathrm{AF}$ & Atrial fibrillation \\
\hline APA & Action potential amplitude $(\mathrm{mV})$ \\
\hline $\mathrm{APD}_{20}$ & $\begin{array}{l}\text { Action potential duration at } 20 \% \text { of repolari- } \\
\text { zation }(\mathrm{ms})\end{array}$ \\
\hline $\mathrm{APD}_{50}$ & $\begin{array}{l}\text { Action potential duration at } 50 \% \text { of repolari- } \\
\text { zation }(\mathrm{ms})\end{array}$ \\
\hline $\mathrm{APD}_{90}$ & $\begin{array}{l}\text { Action potential duration at } 90 \% \text { of repolari- } \\
\text { zation }(\mathrm{ms})\end{array}$ \\
\hline $\mathrm{dV} / \mathrm{dt}_{\max }$ & Maximum rate of depolarization $\left(\mathrm{Vs}^{-1}\right)$ \\
\hline MAD & $\begin{array}{l}\text { Maximum area under ROC curve-based } \\
\text { discretization }\end{array}$ \\
\hline $\mathrm{PLT}_{20}$ & $\begin{array}{l}\text { "Plateau potential" defined as the mean poten- } \\
\text { tial }(\mathrm{mV}) \text { in the time window between } 20 \% \text { of } \\
\mathrm{APD}_{90} \text { plus } 5 \mathrm{~ms}\end{array}$ \\
\hline RIMARC & $\begin{array}{l}\text { Ranking instances by maximizing the area } \\
\text { under the ROC curve }\end{array}$ \\
\hline RMP & Resting membrane potential (mV) \\
\hline & Receiver operating characteristics \\
\hline & Sinus rhythm \\
\hline
\end{tabular}

\section{Introduction}

During open-heart surgery, the outer rim of the right atrial appendage is removed in order to introduce the tubing for connecting the patient to the extracorporeal circulation. Most patients agree that these tissue samples are made available for basic research rather than being disposed. Therefore, right atrial tissue can be studied ex vivo for its basic electrophysiological properties.

Action potentials (APs) recorded in right atrial tissue from patients in sinus rhythm (SR) exhibit a characteristic spikeand-dome morphology that changes into a triangular form due to electrical remodeling in chronic atrial fibrillation (AF) $[4,19,22]$. Many studies have reported in silico modeling of atrial repolarization in order to understand the complex processes which underlie AF-induced changes [6, 7, 15]. Despite the clear association of AP shape with the patient's preoperative rhythm status, the variability in individual AP morphology is large and does not always allow a clear distinction between SR and AF. Besides preoperative rhythm status, other demographic and clinical factors could have an

H. A. Güvenir

Department of Computer Engineering, Bilkent University,

Ankara, Turkey influence on AP shape. Therefore, most published experimental studies with human atrial tissue also include additional information on the patients' age, height, body weight, concomitant diseases, basal hemodynamic parameters, pre-surgical medication, etc. (e.g., [5, 23, 24] ). However, cohort sizes are usually quite small ( $<50$ patients) so that weak influences of accompanying clinical conditions cannot be ruled out.

In the context of our electrophysiological interest in $\mathrm{AF}$ pathophysiology, we have accumulated a large data pool of human right atrial action potential recordings during the past 8 years. From all patients, we have also collected clinical parameters from the electronic (scanned) patient files. Instead of using conventional statistics to find associations between AF and electrophysiological and clinical features, we used a machine-learning approach for risk prediction. In this context, the term "risk prediction" is used in a mathematical sense of correct classification and is not related to the clinical prediction of risk of getting a disease based on empirically observed risk factors for that disease.

The general objective of all machine-learning approaches can be stated as non-trivial extraction of previously unknown, however, hypothesis-generating premises, rules and relations from large data sets. Aligned with this particular objective, our study incorporated a machinelearning algorithm ranking instances by maximizing the area under the ROC curve (RIMARC) [10] instead of a multivariate statistical approach.

The RIMARC algorithm was applied to a large data set of ex vivo recorded human right atrial APs combined with retrospectively collected general clinical parameters. Therefore, the aim of our study was to test whether the rules learned by this algorithm could be used for an accurate classification of a patient's rhythm status.

\section{Methods}

Data included in this study were collected in the period from January 2006 to February 2014. The study conforms to the Declaration of Helsinki and was approved by the ethics committee of Dresden University of Technology (No. EK790799). Each patient gave written informed consent. Right atrial appendages were obtained from 221 patients with SR and 158 patients with chronic $\mathrm{AF}$ at the time of open-heart surgery. The criteria for a patient to be considered in permanent $A F$ were history of ECG-documented $A F$ and symptoms of AF for $\geq 6$ months. Patients with paroxysmal or intermittent AF were excluded. Demographic and clinical data of the patients are summarized in Table 1 and are comparable to the characteristics published previously $[6,7,15]$. The MannWhitney $U$ test was applied for a complete statistical analysis of all features between the SR and AF group of patients, the results are provided in supplementary Table 1 . 
Table 1 Patient characteristics

\begin{tabular}{|c|c|c|}
\hline & SR & $\mathrm{cAF}$ \\
\hline Patients, $n$ & 221 & 158 \\
\hline Gender, $\mathrm{m} / \mathrm{f}(\mathrm{m} \%)$ & $156 / 65(70.6 \%)$ & $94 / 64(59.1 \%)$ \\
\hline Age (years) & $67.8 \pm 0.68$ & $72.9 \pm 0.57 * * * *$ \\
\hline Height (m) & $1.70 \pm 0.01$ & $1.69 \pm 0.01$ \\
\hline Weight $(\mathrm{kg})$ & $79.5 \pm 0.91$ & $79.8 \pm 1.15$ \\
\hline Body mass index $\left(\mathrm{kg} / \mathrm{m}^{2}\right)$ & $27.4 \pm 0.25$ & $27.6 \pm 0.37$ \\
\hline $\operatorname{LVEF}(\%)[n \text { patients }]^{\mathrm{a}}$ & $54.6 \pm 0.92[194]^{\mathrm{a}}$ & $52.6 \pm 0.92[156]$ \\
\hline $\begin{array}{l}\mathrm{LVEDP}(\mathrm{mm} \mathrm{Hg})[n \\
\text { patients }]\end{array}$ & $17.0 \pm 0.92[76]$ & $16.0 \pm 0.69[81]$ \\
\hline LAD $(\mathrm{mm})[n$ patients $]$ & $42.2 \pm 0.43[156]$ & $51.3 \pm 0.66[145]^{* * * *}$ \\
\hline LVEDD $(\mathrm{mm})[n$ patients $]$ & $49.9 \pm 0.62[156]$ & $51.2 \pm 0.67[134]$ \\
\hline IVS $(\mathrm{mm})[n$ patients $]$ & $12.8 \pm 0.18[150]$ & $12.9 \pm 0.16[132]$ \\
\hline LVPW $(\mathrm{mm})[n$ patients $]$ & $12.2 \pm 0.17[146]$ & $12.4 \pm 0.16[131]$ \\
\hline Bypass, $n(\%)$ & $141(55.1 \%)$ & $24(10.7 \%)^{\dagger}$ \\
\hline Valve replacement $(n)$ & $71(27.7 \%)$ & $155(69.2 \%)^{\dagger}$ \\
\hline $\begin{array}{l}\text { Bypass plus valve replace- } \\
\text { ment }(n)\end{array}$ & $44(17.2 \%)$ & $44(19.6 \%)$ \\
\hline Other surgery & 0 & $1(0.4 \%)$ \\
\hline $\begin{array}{l}\text { Hypertension, } n \text { (yes, no, } \\
\text { no inform.) })^{\mathrm{b}}\end{array}$ & $180,14,27$ & $150,5,3$ \\
\hline Diabetes $(n)$ & $80,140,1$ & $64,94,0$ \\
\hline Hyperlipidemia $(n)$ & $151,43,27$ & $108,46,4$ \\
\hline Digitalis $(n)$ & $6,188,28$ & $57,92,4^{\dagger}$ \\
\hline ACE inhibitors $(n)$ & $131,64,26$ & $93,57,8$ \\
\hline AT1 blockers $(n)$ & $40,153,28$ & $45,103,10$ \\
\hline$\beta$-Blockers $(n)$ & $170,25,25$ & $132,21,5$ \\
\hline Dihydropyridines $(n)$ & $36,157,28$ & $35,114,9$ \\
\hline Diuretics $(n)$ & $90,103,28$ & $104,45,9^{\dagger}$ \\
\hline Nitrates $(n)$ & $31,162,28$ & $19,130,9$ \\
\hline Lipid-lowering drugs $(n)$ & $132,61,28$ & $81,68,9$ \\
\hline
\end{tabular}

$S R$ sinus rhythm, $c A F$ chronic atrial fibrillation ( $\geq 6$ months), $m$ male, $f$ female; $L V E F$ left ventricular ejection fraction, $L V E D P$ left ventricular end-diastolic pressure, $L A D$ left atrial diameter, $L V E D D$ left ventricular end-diastolic diameter, IVS interventricular septum, $L V P W$ left ventricular posterior wall, $A C E$ angiotensin-converting enzyme, $A T 1$ angiotensin- type 1 receptor, $\beta$-blockers, $\beta$-adrenoceptor blockers $* * * * P<0.0001, \mathrm{AF}$ versus SR from Student's two-tailed, unpaired $t$ test $\dagger$ Mann-Whitney $U$ test, null-hypothesis rejected (with significance 0.001 and confidence interval $95 \%$ )

a Values in square brackets indicate number of patients for which values are available

b The categories "yes," "no," and "no information" apply to the remaining 11 features

\subsection{Action potential measurements}

Small pieces of human right atrial appendages were transported to the laboratory in a special $\mathrm{Ca}^{2+}$-free transport solution at $20-25^{\circ} \mathrm{C}$, composition in $\mathrm{mM}$ : $100.0 \mathrm{NaCl}, 10.0$ $\mathrm{KCl}, 1,2 \mathrm{KH}_{2} \mathrm{PO}_{4}, 5.0 \mathrm{MgSO}_{4}$, taurin $50 \mathrm{mM}$, MOPS $5 \mathrm{mM}$, $30 \mathrm{mM}$ butanedionemonoxime (BDM), pH $7.0\left(20-25{ }^{\circ} \mathrm{C}\right)$.
Either free-running trabeculae or trabeculae together with attached atrial wall were dissected and mounted on the bottom of a 5-ml organ bath perfused with $50 \mathrm{ml}$ of recirculating, oxygenized Tyrode's solution at a flow rate of $7 \mathrm{ml} /$ min at $36 \pm 1{ }^{\circ} \mathrm{C}$ (composition in $\mathrm{mM}$ : $126.7 \mathrm{NaCl}, 0.42$ $\mathrm{NaH}_{2} \mathrm{PO}_{4}, 22 \mathrm{NaHCO}_{3}, 5.4 \mathrm{KCl}, 1.8 \mathrm{CaCl}_{2}, 1.5 \mathrm{MgCl}_{2}$, pH 7.4 when equilibrated with $5 \% \mathrm{CO}_{2}$ in $\mathrm{O}_{2}$ ). Preparations were electrically stimulated at a single constant rate of $1 \mathrm{~Hz}$ with isolated square-wave stimuli of $1 \mathrm{~ms}$ duration, two times threshold intensity. Transmembrane potentials were recorded with glass microelectrodes filled with $2.5 \mathrm{M}$ $\mathrm{KCl}$. Tip resistances of the electrodes were between 20 and $80 \mathrm{M} \Omega$. Both timing of the driving stimuli and preprocessing of the transmembrane potential responses were carried out with a computer-aided AP recording system.

The trabeculae were used for various different experiments; however, each experiment was preceded by a 60-min equilibration period during which the preparations were allowed to stabilize and residual BDM was completely washed out. At the end of the equilibration period, 10 consecutive APs were averaged and analysed for the following parameters (Fig. 1): resting membrane potential, RMP (mV), action potential amplitude, APA $(\mathrm{mV})$, action potential duration at 20,50 , and $90 \%$ of repolarization $\left(\mathrm{APD}_{20}, \mathrm{APD}_{50}\right.$ and $\mathrm{APD}_{90}$ in ms), maximum rate of depolarization, $\mathrm{dV} / \mathrm{dt}_{\max }(\mathrm{V} / \mathrm{s})$, and the "plateau potential" $\left(\mathrm{PLT}_{20}\right)$ defined as the mean absolute membrane potential $(\mathrm{mV})$ in a 5-ms window starting from $20 \%$ of $\mathrm{APD}_{90}$.

The clinical parameters chosen for our data set are demographic characteristics such as age, weight, body mass index (BMI), etc., clinical conditions associated with heart disease, hemodynamic parameters, and medication. Routine methods and clinical laboratory tests were used for assessment of clinical parameters. For example, left atrial or left ventricular enddiastolic diameter, right ventricular systolic pressure, left ventricular end-diastolic pressure, left ventricular end-diastolic diameter, ejection fraction, cardiomyopathy, left ventricular posterior wall thickness, interventricular septum thickness, mitral or aortic valve insufficiency, aortic stenosis, and patent foramen ovale were determined with trans-thoracal or transesophageal echocardiography. Right heart catheterization was required for diagnosis of pulmonary hypertension and sometimes for measuring right ventricular systolic pressure. Right coronary artery stenosis and number of occluded vessels were diagnosed by coronary angiography. Chronic pulmonary obstructive disease (CPOD) was quantified with spiro-ergometric methods. The complete list of all electrophysiological and clinical parameters is given in Table 2.

\subsection{Data set}

All patients who agreed to participate in this study were anonymized, but had to be assigned a consecutive code 


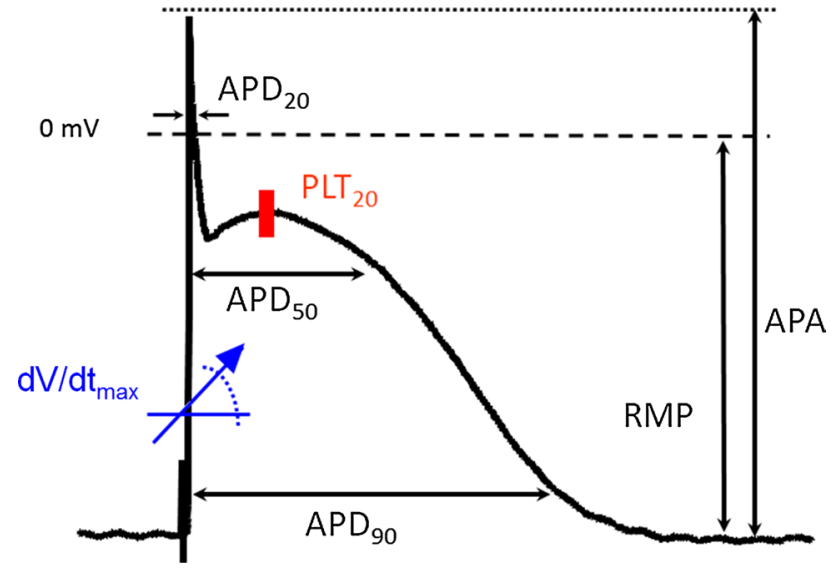

Fig. 1 Action potential from a patient in sinus rhythm to illustrate analysis of action potential parameters. APA action potential amplitude $(\mathrm{mV}) ; R M P$ resting potential $(\mathrm{mV}), \mathrm{APD}_{20}, \mathrm{APD}_{50}$, and $\mathrm{APD}_{90}$, action potential duration at 20,50 , and $90 \%$ of repolarization (in $\mathrm{ms}), \mathrm{dV} / \mathrm{dt}_{\max }$, maximum rate of depolarization $\left(\mathrm{Vs}^{-1}\right)$, and the "plateau potential" defined as the mean absolute membrane potential $(\mathrm{mV})$ in the time window between $20 \%$ of $\mathrm{APD}_{90}$ plus $5 \mathrm{~ms}\left(\mathrm{PLT}_{20}\right)$

number known only to the project leader. This number was used to identify the medical file for collection of available clinical parameters. Patients for whom no clinical data was available were eliminated.

The data set comprised of a total of 480 instances from 379 patients (instances): 452 instances from 351 patients were used for training the algorithm and 28 instances from 28 patients were used for testing. The latter instances were assigned to the testing group, because they were collected after an arbitrary deadline we set for the learning group. In the training data set, there were $214 \mathrm{AF}$ and $238 \mathrm{SR}$ instances, and in the test data set, there were $10 \mathrm{AF}$ and 18 SR instances, and for some patients, action potentials were measured in more than one experiment and were therefore treated as separate instances. This is an accepted approach in machine learning.

All instances are labeled as AF (positive, "P") or SR (negative, "N"). A total of 62 features (seven AP parameters and 56 clinical parameters) were used. Of the features, 24 were numerical (continuous) and 38 were categorical. In both groups, some clinical parameters were missing; the percentage of missing values was $27 \%$ for the training and $2 \%$ for the test data set.

\subsection{Calculations}

\subsubsection{Training phase}

As a machine-learning algorithm, RIMARC is used to distinguish the features and their particular values (or value ranges) that are indicators of AF. This algorithm has been designed to maximize the area under the ROC curve
(AUC). It learns a ranking function, which is a linear combination of nonlinear scoring functions and is learned for each feature. The RIMARC algorithm first discretizes each continuous feature using a maximum area under ROC curve-based discretization (MAD2C) algorithm [17]. For example, the numerical feature $\mathrm{APD}_{90}$ is converted into a categorical feature by partitioning the range of all values, e.g., 140-467 ms, into sub-ranges, e.g., 140-192.5, 192.5205.5, 205.5-229.5, 229.5-243.5, 243.5-247.5 ms, and so on. Thus, each numerical feature is converted into a categorical one by assigning a label to each of these sub-ranges. All values of a numerical feature, e.g., $\mathrm{APD}_{90}$, are replaced by labels corresponding to the sub-ranges in the data set. For example, an $\mathrm{APD}_{90}$ value of $234 \mathrm{~ms}$ will be replaced by the label "229.5. 243.5." It is shown that probability can be used as scoring function for achieving the maximum AUC for a single categorical feature [10]. In this context, probability is the ratio of number of AF cases divided by the number of all cases in corresponding categorical value in the training data set. For example, the score associated with the label " 229.5 .243 .5 " is $0.8928[=25 /(25+3)]$, since there are $25 \mathrm{AF}$ and three SR cases.

For each feature, the instances in the data set are sorted by the corresponding scoring function (probability). The ROC curve (Fig. 2) is constructed, labeling the AF patients as "P" and SR patients as " $\mathrm{N}$ " and calculating true positive rate (TRP) and false positive rate (FPR) of the instances within the class labels (ranges of values) obtained by the discretizing process to yield the maximum area under the curve. The data set is sorted in the order of the score assigned to the ranges of values, and TPR and FPR are calculated in a cumulative manner by working through the sorted list of instances one by one. Mathematically, TPR is the number of true positive instances divided by number of all instances marked as positive, and FPR is the number of false positive instances divided by number of all negative instances in the data set. The area under the ROC curve (AUC) is an indicator of the quality of the ranking; the higher the AUC value, the better the ranking is.

The discretization with MAD2C algorithm [23] optimizes the AUC. Figure 2 illustrates ROC curves for the feature $\mathrm{APD}_{90}$, as a continuous feature (Fig. 2a) and as a discretized feature (Fig. 2b). Without any discretization, the AUC is 0.950 , and after the discretization, it slightly increases to 0.957. This increment in the value of AUC is attributed to the objective function of MAD2C algorithm that aims to yield the maximum AUC by discretizing the continuous range of a feature into optimal discrete ranges.

The AUC value can be used to compute the predictive weight of a feature to classify a patient as AF or SR. The RIMARC algorithm computes the weight of a feature $f$ as $w_{f}=2(A U C(f)-0.5)$, where $A U C(f)$ is the AUC obtained for the feature $f$. If the AUC is equal to 1 , classification is 
Table 2 List of features ordered according to predictive weight

\begin{tabular}{|c|c|c|c|}
\hline Feature name & Predictive weight & Feature name & Predictive weight \\
\hline $\mathrm{APD}_{90}(\mathrm{~ms})$ & 0.9135 & $\mathrm{dV} / \mathrm{dt}_{\max }(\mathrm{V} / \mathrm{s})$ & 0.1976 \\
\hline $\mathrm{APD}_{20}(\mathrm{~ms})$ & 0.8622 & Aortic valve insufficiency & 0.1936 \\
\hline Left atrial end-diastolic diameter (mm) & 0.7513 & Body mass index & 0.1783 \\
\hline $\mathrm{APD}_{50}(\mathrm{~ms})$ & 0.7475 & Patent foramen ovale & 0.1691 \\
\hline $\mathrm{PLT}_{20}(\mathrm{mV})$ & 0.6157 & Left ventricular ejection fraction (\%) & 0.1616 \\
\hline APA $(m V)$ & 0.5451 & Aortic valve insufficiency Grade I-II & 0.1545 \\
\hline Pulmonary hypertension & 0.5309 & Left ventricle end-diastolic diameter (mm) & 0.1513 \\
\hline Surgical procedure & 0.4943 & Intraventricular septum thickness at diastole (mm) & 0.1338 \\
\hline QT-time (ms) & 0.4515 & Aortic Stenosis & 0.1244 \\
\hline RMP (mV) & 0.4272 & Left ventricular wall thickness at diastole (mm) & 0.1205 \\
\hline Number of occluded vessels & 0.4181 & Weight $(\mathrm{kg})$ & 0.1163 \\
\hline Creatinine & 0.4106 & Lipid-lowering drugs & 0.1100 \\
\hline Glomerular filtration rate (GFR) & 0.4104 & Sex & 0.1082 \\
\hline Right ventricular systolic pressure $35-54 \mathrm{mmHg}$ & 0.4061 & Nicotine & 0.1035 \\
\hline Mitral valve insufficiency Grade III & 0.4031 & AT1 blocker & 0.0889 \\
\hline Mitral valve insufficiency & 0.3693 & Left ventricular hypertrophy & 0.0824 \\
\hline QTc-time (ms) & 0.3585 & Hyperlipidemia & 0.0639 \\
\hline Diuretic & 0.3459 & Hypertension & 0.0625 \\
\hline Digitalis & 0.3458 & Mitral stenosis Grade II-III & 0.0598 \\
\hline Age (years) & 0.3411 & Aortic valve insufficiency Grade III & 0.0566 \\
\hline Right coronary artery stenosis $>70 \%$ & 0.3395 & Calcium channel blocker & 0.0563 \\
\hline Potassium, serum $(\mathrm{mmol} / \mathrm{l})$ & 0.3337 & Nitrate & 0.0494 \\
\hline Anamnestic or actual decompensation & 0.3245 & Mitral valve insufficiency Grade II & 0.0386 \\
\hline Mitral valve insufficiency Grade I & 0.3228 & Aortic stenosis Grade I-II & 0.0354 \\
\hline Tricuspid valve insufficiency Grade II-III & 0.3072 & Inflammatory process & 0.0243 \\
\hline Aortic stenosis Grade III & 0.2222 & Chronic obstructive pulmonary disease & 0.0213 \\
\hline Left ventricular end-diastolic pressure $(\mathrm{mmHg})$ & 0.2140 & Obstructive sleep apnea syndrome & 0.0203 \\
\hline Heart failure NYHA III & 0.2125 & ACE Inhibitor & 0.0193 \\
\hline Height (m) & 0.2038 & Betablocker & 0.0122 \\
\hline Myocardial infarction & 0.1984 & Diabetes & 0.0059 \\
\hline Right ventricular systolic pressure $>55 \mathrm{mmHg}$ & 0.1976 & Cardiomyopathy & 0.0047 \\
\hline
\end{tabular}

Fig. 2 ROC curves for the feature $\mathrm{APD}_{90}$, as a continuous feature (a) and as a discretized feature (b). Without any discretization, the area under the ROC curve (AUC) is 0.950 (95\% CI, 0.930-0.971) with the standard error of 0.011 under the nonparametric assumption. However, after the discretization with MAD2C algorithm [23], AUC for $\mathrm{APD}_{90}(\mathbf{b})$ increases slightly and becomes 0.957 (95\% CI, 0.938-0.975) with the standard error of 0.009 under the nonparametric assumption

\section{ROC Curve of $A P D_{90}$}
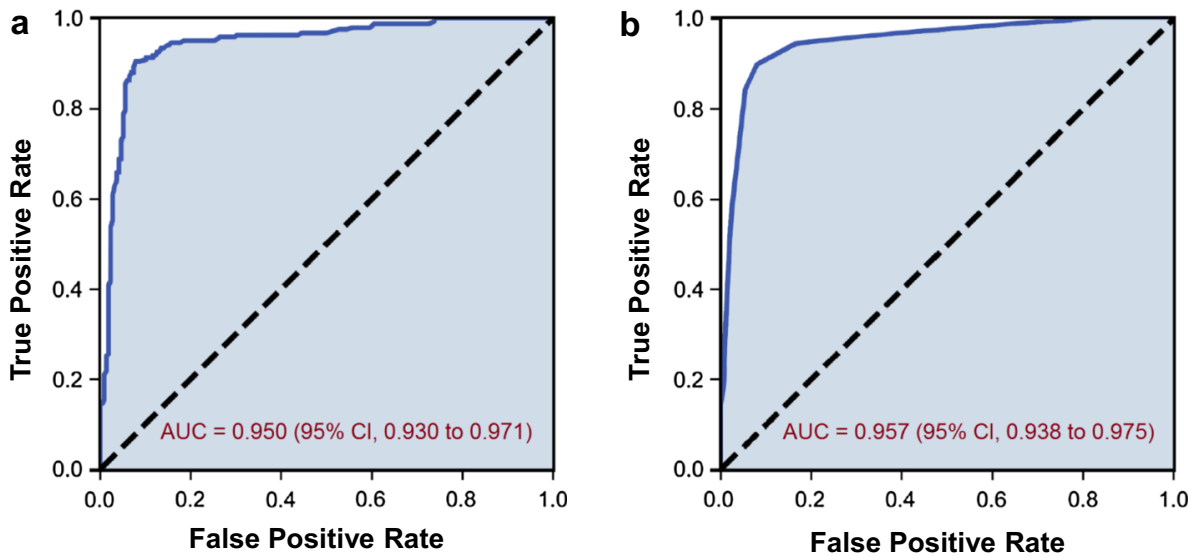
perfect, whereas a value of 0.5 indicates that random guessing has occurred [17].

The training phase is completed by forming one rule for each feature. A rule for a feature contains a score corresponding to each possible value of that feature and the predictive weight for that feature.

\subsubsection{Classification phase}

The rule model learned by the RIMARC algorithm can be used for predicting whether a patient belongs to the AF class (risk score). The risk score of AF for a given query patient $q$ is given by the following formula:

$\operatorname{score}(q)=\frac{\sum_{f} w_{f}^{q} \cdot s_{f}(q)}{\sum_{f} w_{f}^{q}}$

$w_{f}^{q}= \begin{cases}w_{f} & q_{f} \text { is known } \\ 0 & q_{f} \text { is missing }\end{cases}$

Here, $w_{f}$ represents the weight of the feature $f, q_{f}$ is its value, and $s_{f}(q)$ is the score associated with the value of feature $f$ for the query patient $q$. For coping with missing values when computing the risk score, the RIMARC algorithm ignores a feature if the query patient has no value for that feature. As shown in the formula above, $w_{f}^{q}$ is zero, if the value of feature $f$ is missing in patient $q$; otherwise, it is the weight of feature $f$.

All training instances are sorted by the risk score function given above (1). Let $n_{N}$ be the number of negative (SR) patients in the training data set, and let us define a cutoff score as

$$
\begin{aligned}
\operatorname{prediction}(q) & =\left\{\begin{array}{l}
\mathrm{P} \text { if } \operatorname{score}(q)>c \\
\mathrm{~N} \text { otherwise }
\end{array}\right. \\
\text { where } c & =\frac{\operatorname{scors}\left(t_{n N}\right)+\operatorname{scors}\left(t_{n N+1}\right)}{2}
\end{aligned}
$$

Here, $\operatorname{score}\left(t_{n N}\right)$ represents the score of the $n_{N}^{\text {th }}$ training instance. If the risk score function is perfect, all first $n_{N}$ training instances will be negative instances. Vice versa, all instances whose risk score value is greater than $c$ must be positive instances (see Fig. 3). Thus, we predict the class of a query patient as $P$ if its risk score is greater than $c$; negative otherwise.

\section{Results}

\subsection{Electrophysiological recordings}

In our large cohort of human right atrial APs, we confirm the characteristic spike-and-dome (SR) and a more triangular conformation (AF) (Fig. 4). With the exception of maximum upstroke velocity $\mathrm{dV} / \mathrm{dt}_{\max }$, mean values of all

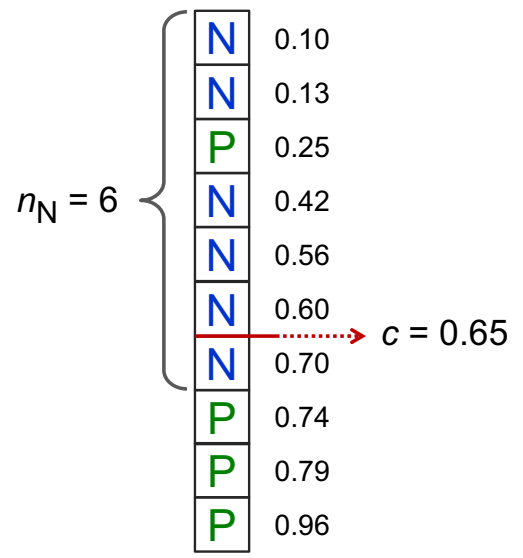

Fig. 3 Illustration to calculate the cutoff score $c$ in a toy data set. All instances are sorted by their individual risk score given by Eq. (1). N (negative instances) represent SR patients; $\mathrm{P}$ (positive instances) represent $\mathrm{AF}$ patients, $\mathrm{n}_{\mathrm{N}}$ is the total number of negative instances, e.g., $n_{\mathrm{N}}=6$ in this data set; cutoff score c is calculated by Eq. (2), i.e., average between risk score of instances six and seven from the top. See text for further details

commonly analysed AP parameters (i.e., $\mathrm{APD}_{90}, \mathrm{APD}_{50}$, $\mathrm{APD}_{20}, \mathrm{PLT}_{20}, \mathrm{APA}, \mathrm{RMP}$ ) were highly significantly different among SR and AF groups. The histograms in Fig. 5 show substantial overlap of value distribution.

\subsection{Rules generated by the RIMARC algorithm}

All electrophysiological and clinical features according to their predictive weights calculated by the RIMARC algorithm are listed in decreasing order of predictive power in Table 2. The rules for all features are given in Supplementary Table 2. Using the MAD2C algorithm, RIMARC automatically divided the total range of the feature $\mathrm{APD}_{90}$ into 11 sub-ranges. For the rule about the feature $\mathrm{APD}_{90}$, our training data set contained 71 instances of $\mathrm{APD}_{90}$ values in the range of 205.5 and $229.5 \mathrm{~ms}$, and $91.5 \%$ were from patients with AF. The risk plot depicted in Fig. 6 demonstrates the nonlinear increase in risk with decreasing values of $\mathrm{APD}_{90}$. Further, the predictive weight of $\mathrm{APD}_{90}$ is 0.9135 which is the highest among all the features.

Left atrial diastolic diameter was divided into 10 subranges. According to this particular rule learned from the training data, all 44 cases, i.e., $100 \%$, with left atrial diameter larger than $55.5 \mathrm{~mm}$, and $90.9 \%$ of the cases with left atrial diameters in the range of $52.5-55.5 \mathrm{~mm}$ had AF. The predictive weight of this feature was 0.7513 .

All patients required cardiac surgery, including valve replacement only, valve replacement plus closure of atrial septal defect (ASD), valve replacement plus coronary artery bypass, and coronary artery bypass only. According to the rule about surgical procedure, there were 215 cases with valve replacement, and $68.4 \%$ had AF. The predictive 
Fig. 4 a Characteristic traces of two human right atrial action potentials representative for tissue from a sinus rhythm (SR) and an atrial fibrillation (AF) patient. b Mean values \pm standard error of the mean, $n$ number of patients; $\sigma_{\mathrm{SR}}$ and $\sigma_{\mathrm{AF}}$, standard deviation for $\mathrm{SR}$ and $\mathrm{AF}$, respectively. See legend of Fig. 1 for explanation of abbreviations a
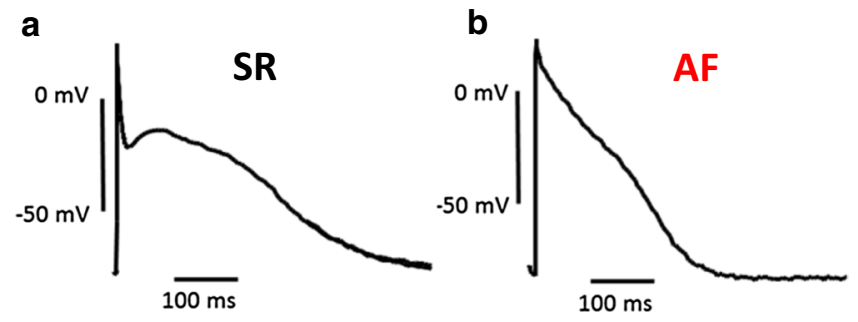

\begin{tabular}{ll|r|c|c|c|c}
\hline & \multicolumn{1}{|c|}{$\begin{array}{l}\text { SR ( }=\mathbf{2 3 8}) \\
{[\mathrm{Cl}=\mathbf{0 . 9 9 9}]}\end{array}$} & $\boldsymbol{\sigma}_{\mathrm{SR}}$ & $\begin{array}{c}\text { AF }(\mathrm{n}=\mathbf{2 1 4}) \\
{[\mathrm{Cl}=0.999]}\end{array}$ & $\boldsymbol{\sigma}_{\mathrm{AF}}$ & $\begin{array}{c}\text { Sig (2-tailed) } \\
{[\mathrm{Cl}=\mathbf{0 . 9 9 9}]}\end{array}$ \\
\hline $\mathbf{A P D}_{\mathbf{9 0}}$ & $\mathrm{ms}$ & $317.41 \pm 9.33$ & 43.19 & $217.45 \pm 8.15$ & 35.74 & $<0.001$ \\
\hline $\mathbf{A P D}_{\mathbf{5 0}}$ & $\mathrm{ms}$ & $138.09 \pm 9.75$ & 45.14 & $100.41 \pm 6.31$ & 27.68 & $<0.001$ \\
$\mathbf{A P D}_{\mathbf{2 0}}$ & $\mathrm{ms}$ & $7.22 \pm 1.83$ & 8.48 & $29.41 \pm 4.15$ & 18.17 & $<0.001$ \\
$\mathbf{P L T}_{\mathbf{2 0}}$ & $\mathrm{mV}$ & $-16.28 \pm 1.40$ & 6.49 & $-5.09 \pm 2.57$ & 11.26 & $<0.001$ \\
$\mathbf{A P A}$ & $\mathrm{mV}$ & $94.95 \pm 1.52$ & 7.07 & $101.56 \pm 1.76$ & 7.71 & $<0.001$ \\
$\mathbf{R M P}$ & $\mathrm{mV}$ & $-73.98 \pm 0.86$ & 3.99 & $-76.85 \pm 0.83$ & 3.61 & $<0.001$ \\
$\mathbf{d V} / \mathbf{d t}_{\text {max }}$ & $\mathrm{V} / \mathrm{s}$ & $219.44 \pm 14.65$ & 67.85 & $231.56 \pm 16.51$ & 72.42 & 0.067 \\
\hline
\end{tabular}

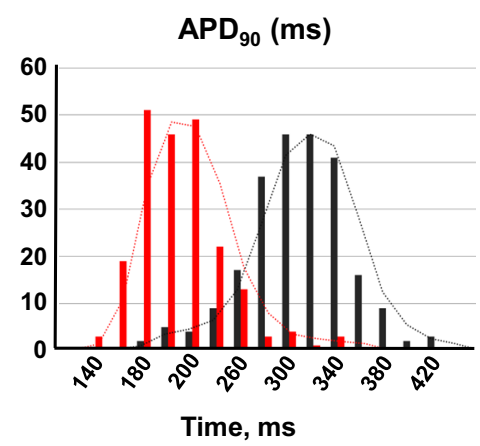

APA $(m V)$

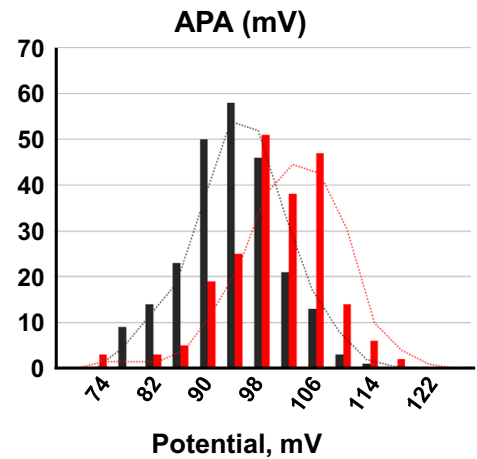

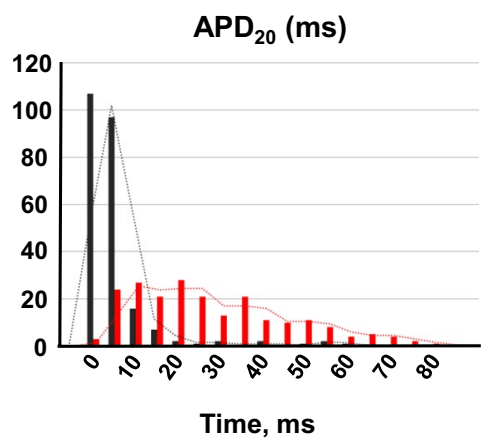

RMP (mV)

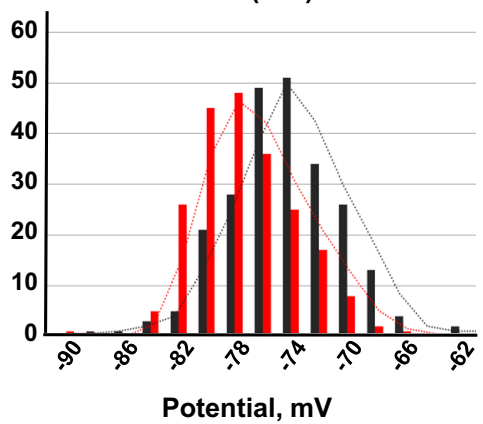

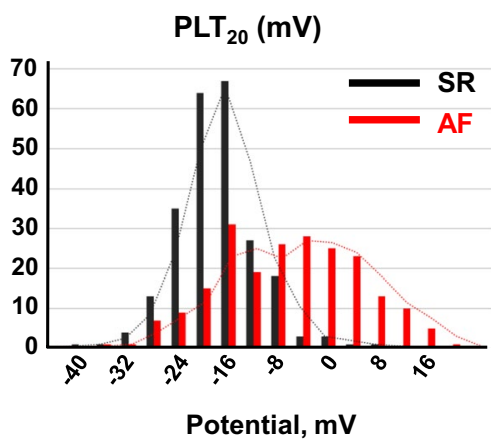

$d V / d t_{\max }(\mathrm{V} / \mathrm{s})$

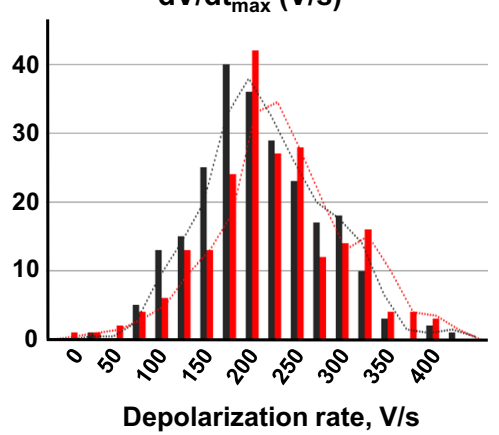

Fig. 5 Histograms of selected action potential parameters from SR (black columns) and $\mathrm{AF}$ trabeculae (red columns): $\mathrm{APD}_{20}, \mathrm{APD}_{90}$, $\mathrm{PLT}_{20}, \mathrm{APA}, \mathrm{RMP}, \mathrm{dV} / \mathrm{dt}_{\max }$. Ordinate: number of preparations with

weight is 0.4943 making it the eighth most effective feature for AF classification.

According to the rule about diabetes, $47 \%$ of the 170 cases with diabetes in the training data set had AF. On the other hand, the same percentage of 281 cases without the ranges indicated by the bins (abscissa). Please note the huge variability within the SR and AF groups

diabetes had AF, as well. The predictive weight of diabetes is 0.0059 , which is second to the least predictive feature.

The rule learned for age discretizes the total age range into nine sub-ranges. For the sub-range 65.5-67.5 years, $50 \%$ of the 40 cases in the training data set had AF. The 

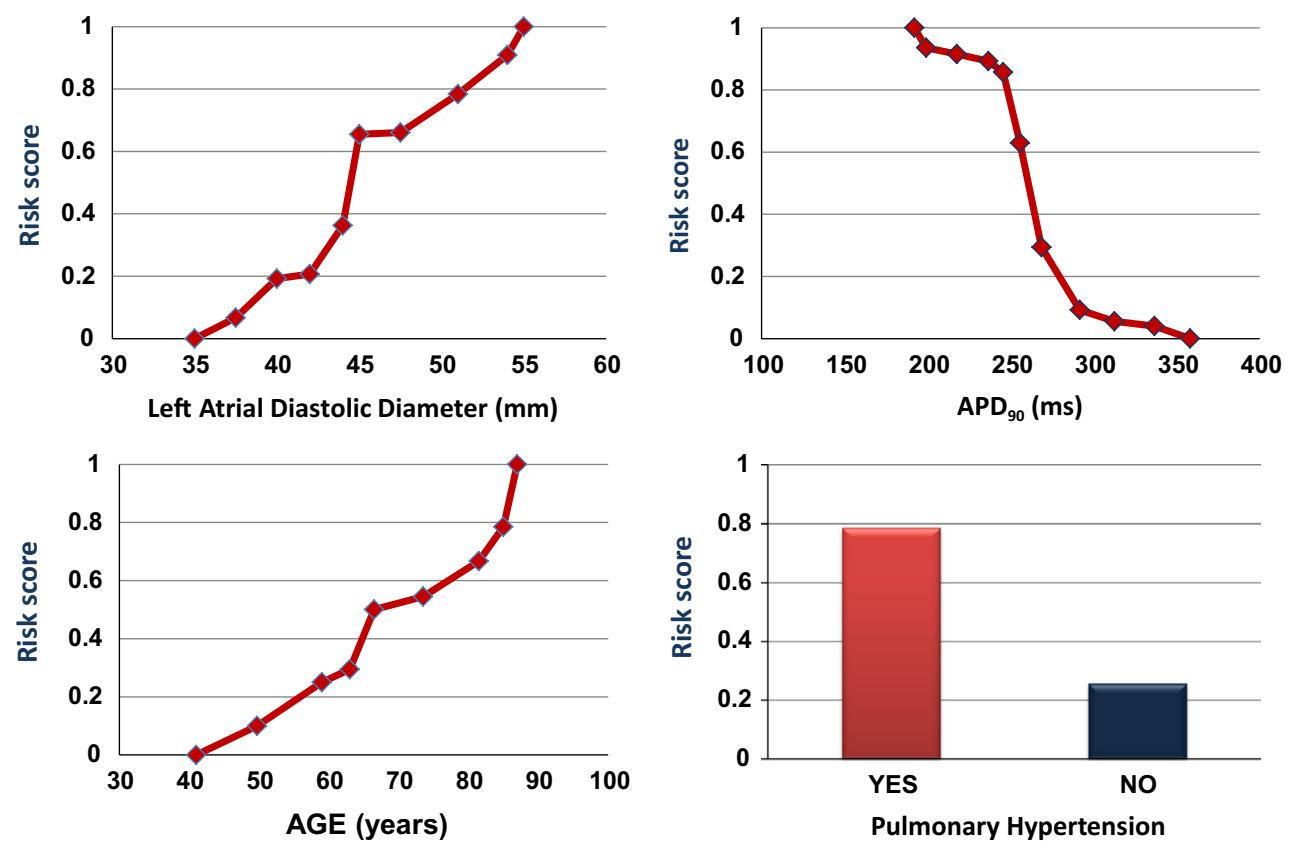

Fig. 6 Plots of risk scores generated from the rules learned by RIMARC for APD 90 , left atrial diameter, age, and pulmonary hypertension. The risk score is the probability of a patient within the discretized sub-ranges to belong to the AF group

Pat. 3832 (AF)

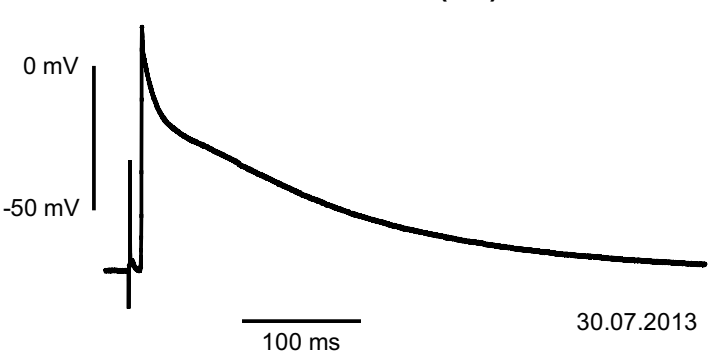

Pat. 3850 (SR)

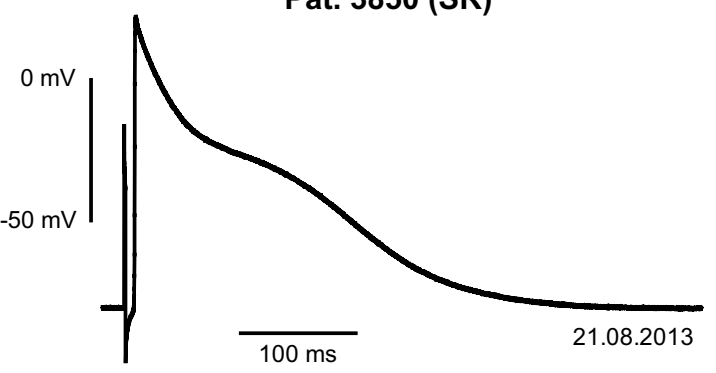

Fig. 7 Action potentials from the two patients $(3,832,3,850)$ misclassified in the test data set. According to the clinical records, patient 3,832 had been in AF but was classified as SR, and patient 3,850 had been in SR but was classified as AF

predictive weight of age is computed to be 0.3411 . The rule demonstrates that the probability of AF increases monotonically as the age increases (see also risk plot in Fig. 6).

\subsection{Testing phase}

In order to validate the rules learned by the RIMARC algorithm, we reserved data from a separate group of 28 patients. The risk scores for these patients are computed according to Eq. 1 which is based on the rules learned in the training phase. The rhythm values for 28 test instances are predicted by Eq. 2. At the end of the training phase, the cutoff score was found to be 0.548 . Those instances with risk score greater than 0.548 are predicted to be AF.
Two out of 28 test instances were misclassified, i.e., one SR patient was predicted to belong to the AF group, and one AF patient was predicted to belong to the SR group. In fact, the shapes of the APs recorded in these two misclassified patients (see Fig. 7) were not typical for their respective rhythm group. The classification accuracy was $0.93(26 / 28)$ when all features were used. The increase in accuracy with increasing numbers of features in the order of their predictive weight (predictive weight or "risk value" in Table 2) is illustrated in Fig. 8. In fact, the six features with the highest weight, i.e., $\mathrm{APD}_{90}, \mathrm{APD}_{20}$, Left atrial diastolic diameter, $\mathrm{APD}_{50}, \mathrm{PLT}_{20}$, and APA, were enough to obtain 0.93 classification accuracy. Excluding all seven AP features from the training data set, classification accuracy was still $0.71(20 / 28)$. 


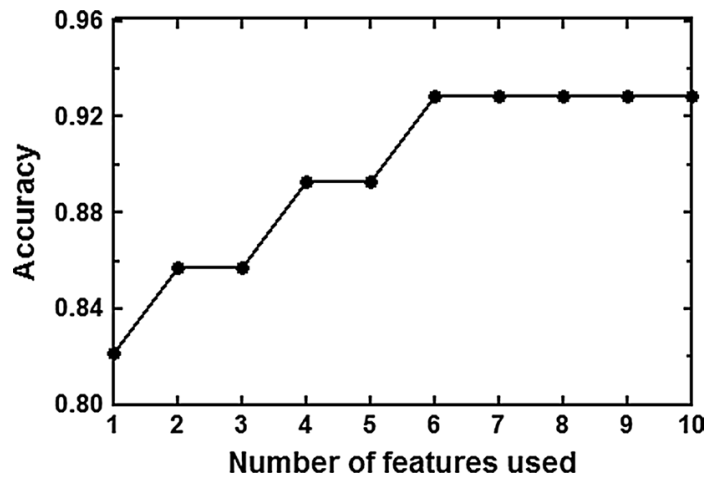

Fig. 8 Accuracy of rhythm classification in the test data set with increasing numbers of features used for the training phase (top 10 features of Table 1, i.e., $\mathrm{APD}_{90}, \mathrm{APD}_{20}$, Left atrial diastolic diameter, $\mathrm{APD}_{50}, \mathrm{PLT}_{20}, \mathrm{APA}$, pulmonary hypertension, surgical procedure, QT-time, and RMP. Please note that the top six features with the highest predictive weight provided total accuracy

\section{Discussion}

In the present study, we have used a large ex vivo electrophysiological data set from human atrial biopsies as well as clinical information for training a machine-learning algorithm to establish a ranking order of features by predictive value for preoperative AF. The major findings were (i) $\mathrm{APD}_{90}, \mathrm{APD}_{20}$, and left atrial diastolic diameter had the highest predictive values; (ii) pulmonary hypertension ranked on place seven for predictive weight, (iii) the clinical features "diabetes" and "treatment with $\beta$-blockers" had lowest predictive values, and (iv) challenging the model with a test data set yielded high accuracy in risk prediction.

\subsection{Action potentials}

In chronic AF, the APD is significantly shorter than in SR due to electrical remodeling $[4,25]$, although there is a substantial overlap in value distributions between the two groups (see Fig. 5). The variability in AP morphology, regardless of rhythm status, confirms reports in the literature $[1-3,9,13,19,21,24]$. Despite this variability, APD $_{90}$ and $\mathrm{APD}_{20}$ have the highest predictive values in our model. Based on our results, the plateau potential PLT $_{20}$ has more negative values in SR than in AF; its predictive weight is 0.6157 for a patient to be in AF.

\subsection{Clinical features}

Atrial fibrillation is characterized by highly irregular excitatory activity that can be triggered by ectopic pacemakers often located in the left atrium and the pulmonary veins [11]. Dilation of the left atrium enhances the likelihood of ectopic activity and stability of re-entrant circuits, and hence susceptibility to AF $[8,16]$, providing a pathophysiological link between mitral valve stenosis or congestive heart failure and increased risk of AF. Thus, the high predictive weight of the feature left atrial diameter in our data set was not surprising. Pulmonary hypertension, however, is not among the well-established risk factors for AF [20], yet its predictive weight (0.5309) ranked on position seven among the 62 predictive features in our data set. In the general population, old age is associated with an increase in pulmonary artery pressure [18], and an expert panel on pulmonary hypertension has recently suggested to introduce a new clinical entity of "pulmonary hypertension of old age" (Ali Oto, personal communication). Age is also a known risk factor for $\mathrm{AF}$ (for review see [14] ). In our data set, the rule for age provided by the RIMARC algorithm demonstrates continuously increasing risk score for AF with increasing age, suggesting that there might be an association between pulmonary hypertension and AF, illustrating the hypothesis-generating aspect of our approach.

On the other hand, age had a rather low predictive weight for AF in our data set, despite the fact that old age is an accepted risk factor for AF [14]. This seeming discrepancy is possibly due to the particular set of patients studied, because patients were not primarily selected for heart rhythm, but, for obvious reasons, only patients requiring open-heart surgery could be included. Similarly, patient selection may also explain the low predictive weight of diabetes and drug treatment, because these two features are similar in all severely ill cardiac patients.

\subsection{Machine-learning algorithm}

The RIMARC algorithm is chosen to be applied in our study due to its several prominent characteristics. First of all, RIMARC achieves comparably high AUC (Area Under the ROC Curve) values, which is the ultimate measure of the precision of a classifier. There are benefits to using such a measure, since AUC has important characteristics, such as insensitivity to class distribution and cost distributions. Another benefit of the AUC is that it has an intuitive probability interpretation: The AUC represents the probability that a randomly chosen positive instance is correctly assigned a higher rank value than a randomly selected negative instance. Therefore, the weights of the features are proportional to their ability to independently classify the instances. Since the RIMARC algorithm uses all available feature values and ignores the missing ones, it is robust to missing feature values. For this reason, it is ideally situated as a valid machine-learning method with experimental and clinical data sets comprising substantial amount of missing values due to the difficulties in data collection and consolidation. In addition to this, being a nonparametric method, RIMARC does not require tuning of any parameters to 
achieve the best performance. Nonparametric methods are preferable when parametric or distributional assumptions about the underlying populations of the data sets are arguable [12].

Most importantly, the RIMARC algorithm learns even nonlinear risk score functions of the values of a given feature. The ranking score functions are in a human readable form that can be easily interpreted by domain experts. The feature weights learned help the experts to determine how they affect the ranking. In this sense, the output of RIMARC is inherently a Decision Support System, which can be used to estimate the risk of a new instance given the model learned from the data set.

\subsection{Study limitations}

There are several limitations of our study, including selection of patients and retrospective collection of clinical data. Criteria for selection of clinical features for this study were chosen not systematically, but on the basis of availability and anticipated relationship in some direct or indirect way to the pathophysiology of AF. They included plain physical parameters, often diagnosed comorbidities in patients with cardiovascular disease, routinely measured hemodynamic parameters, and baseline drug treatment. We could not provide an independent quality control of data, relying solely on the information provided by the patient files. Patients were considered to be in chronic AF when they had symptoms for at least 6 months and ECG documentation of AF on two occasions during this period. Patients with intermittent $\mathrm{AF}$ or patients without any symptoms may have been misclassified if they were in SR at the time of surgery. Since all patients were surgical candidates, some clinical features such as diabetes or treatment with $\beta$-blockers had low predictive values because they occur with similar frequency in severely ill cardiac patients.

\section{Conclusion}

The machine-learning algorithm RIMARC provides a very useful tool for classifying patients according to their preoperative rhythm status ("predicting" rhythm status) with high accuracy from an experimental and clinical set of data. In a clinical setting, this approach may prove useful for finding hypothesis-generating associations between different parameters.

Acknowledgments The authors thank all patients who took part in this study. We gratefully acknowledge the excellent technical assistance of Konstanze Fischer, and the help of Dr. Katrin Ploetze with the logistics of handling patient's files. The authors are grateful for the generous financial support by the European Union (FP7-Health T2-2010-261057 “EUTRAF").

\section{References}

1. Britton OJ, Bueno-Orovio A, Van AK, Lu HR, Towart R, Gallacher DJ et al (2013) Experimentally calibrated population of models predicts and explains intersubject variability in cardiac cellular electrophysiology. Proc Natl Acad Sci USA 110(23):E2098-E2105

2. Bustamante JO, Watanabe T, Murphy DA, McDonald TF (1982) Isolation of single atrial and ventricular cells from the human heart. Can Med Assoc J 126(7):791-793

3. Dawodu AA, Monti F, Iwashiro K, Schiariti M, Chiavarelli R, Puddu PE (1996) The shape of human atrial action potential accounts for different frequency-related changes in vitro. Int $\mathbf{J}$ Cardiol 54(3):237-249

4. Dobrev D, Ravens U (2003) Remodeling of cardiomyocyte ion channels in human atrial fibrillation. Basic Res Cardiol 98(3):137-148

5. Dobrev D, Wettwer E, Himmel HM, Kortner A, Kuhlisch E, Schuler S et al (2000) G-Protein beta(3)-subunit $825 \mathrm{~T}$ allele is associated with enhanced human atrial inward rectifier potassium currents. Circulation 102(6):692-697

6. Dorn A, Krueger MW, Seemann G, Doessel O (2012) Modelling of heterogeneous human atrial electrophysiology. Biomed Tech (Berl) 57(Suppl):1

7. Dossel O, Krueger MW, Weber FM, Wilhelms M, Seemann G (2012) Computational modeling of the human atrial anatomy and electrophysiology. Med Biol Eng Comput 50(8):773-799

8. Eckstein J, Verheule S, de Groot N, Allessie M, Schotten U (2008) Mechanisms of perpetuation of atrial fibrillation in chronically dilated atria. Prog Biophys Mol Biol 97(2-3):435-451

9. Gelband H, Bush HL, Rosen MR, Myerburg RJ, Hoffman BF (1972) Electrophysiologic properties of isolated preparations of human atrial myocardium. Circ Res 30(3):293-300

10. Güvenir HA, Kurtcephe M (2013) Ranking instances by maximizing the area under ROC curve. IEEE Trans Knowl Data Eng 25(10):2356-2366

11. Haissaguerre M, Jais P, Shah DC, Takahashi A, Hocini M, Quiniou $G$ et al (1998) Spontaneous initiation of atrial fibrillation by ectopic beats originating in the pulmonary veins. N Engl J Med 339(10):659-666

12. Hill T, Lewicki P (2005) Statistics: methods and applications. A Comprehensive refeence for science, industry and data mining, 1st. 2005. StatSoft Inc., Tulsa, OK

13. Jakob H, Oelert H, Rupp J, Nawrath H (1989) Functional role of cholinoceptors and purinoceptors in human isolated atrial and ventricular heart muscle. Br J Pharmacol 97(4):1199-1208

14. Kirchhof P, Lip GY, Van Gelder IC, Bax J, Hylek E, Kaab S et al (2012) Comprehensive risk reduction in patients with atrial fibrillation: emerging diagnostic and therapeutic options-a report from the 3rd Atrial Fibrillation Competence NETwork/European Heart Rhythm Association consensus conference. Europace 14(1):8-27

15. Krueger MW, Dorn A, Keller DU, Holmqvist F, Carlson J, Platonov PG et al (2013) In-silico modeling of atrial repolarization in normal and atrial fibrillation remodeled state. Med Biol Eng Comput 51(10):1105-1119

16. Kuijpers NH, Potse M, van Dam PM, ten Eikelder HM, Verheule S, Prinzen FW et al (2011) Mechanoelectrical coupling enhances initiation and affects perpetuation of atrial fibrillation during acute atrial dilation. Heart Rhythm 8(3):429-436

17. Kurtcephe M, Güvenir HA (2013) A discretization method based on maximizing the area under receiver operating characteristic curve. Int J Pattern Recogn Artific Intel 27(Art. No. 1350002):1-26

18. Lam CS, Borlaug BA, Kane GC, Enders FT, Rodeheffer RJ, Redfield MM (2009) Age-associated increases in pulmonary 
artery systolic pressure in the general population. Circulation 119(20):2663-2670

19. Li GR, Nattel S (1997) Properties of human atrial ICa at physiological temperatures and relevance to action potential. Am J Physiol 272(1 Pt 2):H227-H235

20. Rottlaender D, Motloch LJ, Schmidt D, Reda S, Larbig R, Wolny $M$ et al (2012) Clinical impact of atrial fibrillation in patients with pulmonary hypertension. PLoS ONE 7(3):e33902

21. Sanchez C, Bueno-Orovio A, Wettwer E, Loose S, Simon J, Ravens U et al (2014) Inter-subject variability in human atrial action potential in sinus rhythm versus chronic atrial fibrillation. PLoS ONE 9(8):e105897
22. Van Wagoner DR, Nerbonne JM (2000) Molecular basis of electrical remodeling in atrial fibrillation. J Mol Cell Cardiol 32(6):1101-1117

23. Van Wagoner DR, Pond AL, Lamorgese M, Rossie SS, McCarthy PM, Nerbonne JM (1999) Atrial L-type $\mathrm{Ca}^{2+}$ currents and human atrial fibrillation. Circ Res 85(5):428-436

24. Wettwer E, Hala O, Christ T, Heubach JF, Dobrev D, Knaut M et al (2004) Role of $I_{K u r}$ in controlling action potential shape and contractility in the human atrium: influence of chronic atrial fibrillation. Circulation 110(16):2299-2306

25. Workman AJ, Kane KA, Rankin AC (2008) Cellular bases for human atrial fibrillation. Heart Rhythm 5(6 Suppl):S1-S6 